Relations industrielles

Industrial Relations

\title{
Manpower Planning and Programming, by Elmer H. Burack and James W. Walker, Boston, Allyn \& Bacon, Inc., 1972, 452 pp.
}

Jean Sexton

Volume 27, numéro 4, 1972

URI : https://id.erudit.org/iderudit/028350ar

DOI : https://doi.org/10.7202/028350ar

Aller au sommaire du numéro

Éditeur(s)

Département des relations industrielles de l'Université Laval

ISSN

0034-379X (imprimé)

1703-8138 (numérique)

Découvrir la revue

Citer ce compte rendu

Sexton, J. (1972). Compte rendu de [Manpower Planning and Programming, by Elmer H. Burack and James W. Walker, Boston, Allyn \& Bacon, Inc., 1972, 452 pp.] Relations industrielles / Industrial Relations, 27(4), 796-797.

https://doi.org/10.7202/028350ar

Tous droits réservés @ C Département des relations industrielles de l'Université Laval, 1972
Ce document est protégé par la loi sur le droit d'auteur. L'utilisation des services d'Érudit (y compris la reproduction) est assujettie à sa politique d'utilisation que vous pouvez consulter en ligne.

https://apropos.erudit.org/fr/usagers/politique-dutilisation/ 
n'aurait pas d'autre choix que de recommander la totalité des revendications de l'une ou l'autre des parties. Ce mécanisme fait actuellement l'objet d'étude par une commission d'enquête sénatoriale aux Etats-Unis et il est de plus en plus question que le Président Nixon propose qu'elle s'applique au secteur des transports et communications qui a été marqué par des grèves très coûteuses pour l'économie américaine au cours des dernières années.

Enfin, le dernier chapitre analyse différentes techniques de prévention et de solution des conflits telles la médiation, la conciliation, les commissions d'enquête factuelles (Fact Finding Boards), l'arbitrage obligatoire ou volontaire.

Un coup d'œil rapide à la liste des auteurs qui ont collaboré à ce volume permet d'établir hors de tout doute la qualité des ouvrages qu'il contient. Qu'il soit permis de mentionner, outre Neil W. Chamberlain, la collaboration de Arvid Anderson, Charles M. Rehmus, Arnold R. Weber, Sterling D. Spero, Milton Derber, Theodore W. Kheel, Jack Stieber, Jean T. McKelvey. Bref, il s'agit d'un ouvrage indispensable à toute personnes qui veut prétendre connaître quelque chose aux relations du travail dans le secteur public américain.

Jean BOIVIN

Manpower Planning and Programming, by Elmer $H$. Burack and James W. Walker, Boston, Allyn \& Bacon, Inc., 1972, $452 \mathrm{pp}$.

Cet ouvrage possède essentiellement deux caractéristiques: d'abord c'est un recueil d'études (plus de trente cinq). Ensuite il centre son attention sur la planification de main-d'œuvre au niveau micro-économique. «It describes in detail, within the context of technological, social and economic change, approaches for manpower planning and programming in large organizations ». En plus, il est à noter que cet ouvrage s'identifie comme un livre de base pour un cours universitaire, tant au niveau gradué que sousgradué, sur la planification de la maind'oeuvre au niveau de l'entreprise et aussi comme un complément pour un cours sur la direction du personnel.

Tout comme les relations industrielles, cette approche dite de main-d'oeuvre est multi-disciplinaire. A toute fin pratique on en est peut-être arrivé à substituer aux expressions de « relations industrielles » et de «direction du personnel» le qualificatif de main-d'œuvre. L'influence américaine sur ce point, l'impact de l'expression anglaise «manpower et la nouvelle vogue de «main-d'œuvre » peuvent expliquer qu'on étiquette maintenant une réalité assez vieille d'un nouveau nom.

Par contre, ce livre n'est pas un bouquin sur la direction du personnel mais plutôt sur cette partie de la direction du personnel qui se préoccupe de recrutement, de sélection, de promotion, de séparation et de planification. En un mot on se centre plus ici sur la sous-fonction de «staffing 》 de la fonction de direction de personnel, si on peut employer une expression de French.

Essentiellement les auteurs prétendent intégrer l'information éparse déjì existante sur cet aspect et présenter le concept de «manpower planning and programming dans un cadre logique nouveau.

Cet ouvrage comporte quatre parties principales: la première, intitulée «vue générale 》 décrit les récents changements technologiques, sociaux et économiques et leurs implications. En un mot cette partie constitue un arrière-plan qui permet au lecteur de mieux situer le problème. Dans un deuxième temps, on consacre trois chapitres pour expliquer le concept de planification de main-d'œuvre. Cette partie insiste sur des modèles de prévision de main-d'œuvre, sur des modèles de planification et sur des systèmes d'information sur la main-d'oeuvre.

Comme le proposait Vetter (qui malheureusement ne participe pas à cet ouvrage) dans une de ses publications, cette planification doit avoir un impact sur les différentes parties de la sous-fonction de «staffing 》. C'est effectivement ce que Burack et Walker font dans la troisième partie de cet ouvrage. Cependant, cette action qui doit suivre la planification est analysée ici en termes d'approches modernes à la programmation de la main-d'oeuvre. On examine ces approches pour le recrutement, le développement, les plans de carrières, les promotions et les remplacements, surtout le remplacement de cadres.

Enfin, les auteurs se concentrent sur quelques problèmes fondamentaux d'une telle planification de main-d'œuvre. 
Cet ouvrage est sûrement original à plusieurs points de vue. Il a le mérite d'avoir regroupé des noms intéressants à l'intérieur du même volume. Pensons entre autres à Cassell, Rogers, Heneman, Seltzer, Doeringer, Piore, Scoville, Hinrich, Vroom. Une chose peut être regrettable sur ce point, c'est de n'avoir pas inclus un texte de Vetter qui a déjà élaboré un modèle sur le sujet. En plus, cet ouvrage a le mérite de traiter la planification de la main-d'œuvre au niveau micro-économique plus profondément que la grande majorité des ouvrages sur ce point. D'habitude, ce sujet de planification est surtout examiné à l'intérieur d'articles de revues. Un livre examine un sujet plus en profondeur, du moins en principe. Cependant ce livre est essentiellement une collection de textes et peut donc avoir les défauts qu'on attribue généralemcnt aux 《readings ».

Une faiblesse de cet ouvrage, comme dans la grande majorité des ouvrages qui traitent de planification de main-d'œuvre au niveau de l'entreprise, c'est d'oublier un élément de la sous-fonction de 《staffing 》, les départs de la main-d'oeuvre et surtout les départs des travailleurs de production. Quand on traite ce sujet, et c'est relativement rare car on insiste surtout sur les cadres, on insiste surtout sur l'aspect quantitatif. Il serait peut être temps de se préoccuper des licenciements involontaires, pour des raisons imputables à la firme surtout, et ce tant au plan de la planification qu'au plan de l'action à entreprendre pour aider ces travailleurs. Ce sujet n'est sûrement pas nouveau, mais on a tendance à se faire un devoir de l'oublier en pratique.

\section{Jean SEXTON}

\section{Shutdown: The Impact of Plant Shut- down, Extensive Employment Termi- nations and Layoffs on the Workers and the Community, by J.N. Eleen and A.G. Bernardine, Ontario Federa- tion of Labour, Research Department, 1971, 135 pp.}

Voici un document syndical sur un problème qui a fait couler beaucoup d'encre depuis le début du siècle mais qui, malgré les opinions de quelques lunatiques, demeure d'une actualité brùlante. En effet, tous ceux qui s'intéressent aux problèmes des fermetures d'usines, des licenciements collectifs et des mises à pied massives savent que la solution miracle à ces problèmes n'a pas encore été trouvée.

Cet ouvrage est le résultat pratique d'un projet dont le mandat était \& that the OFL undertake to do a study of plant shutdown, plant reduction, termination of employment and extensive layoffs situations in Ontario, their sources and causes, their contribution to the overall unemployment, what effect they have on the economy, ascertain what legislative protection exists, and draft proposals for solution to the problem through legislation, contractual safeguards and other means $\gg$. En fait, un mandat à portée très vaste dont l'exécution complète exigerait beaucoup plus que le travail qui fut en fait accompli.

Pour compléter leur mandat, Eleen et Bernardine ont effectué une enquête couvrant 16,224 travailleurs et 138 compagnies de 25 employés et plus. Quoique leur questionnaire soit inclus en appendice, il n'y a pas assez de détails sur la façon dont l'échantillon a été choisi et sur l'administration du questionnaire. Les auteurs ont choisi de suivre le plan suivant. Après une trop courte introduction (chap. 1 et 2 ) où ils situent superficiellement le problème et présentent leur enquête, les chercheurs résument la situation économique (chap. 3) en rappelant le chômage trop élevé et la dépendance vis-à-vis les Etats-Unis. Vient ensuite l'examen de quatre industries importantes en Ontario (textile, électricité, automobile, acier et mine) et l'examen des réactions de quatre communautés industrielles face aux problèmes ci-haut mentionnés. On retrouve ensuite des titres de chapitres tels le rôle de la filiale dans l'économie ontarienne. l'impact des fermetures d'usines sur les travailleurs et sur les communautés, le rôle de la con. vention collective, le revenu annuel garanti et un examen des législations québecoise, ontarienne et fédérale en cette matière.

Fondamentalement ce livre n'ajoute rien de neuf à la volumineuse littérature déjà existante sur le sujet. Les auteurs, n'ayant pas tenu compte des nombreux travaux en ce domaine, ont été amenés à redécouvrir des choses qui étaient déjà connues. Plusieurs de leurs assertions ne sont pas étayées par des preuves.

Les qualités de cet ouvrage sont très peu nombreuses. On y retrouve un bon chapitre sur les limites de la convention 